\title{
Erratum to: Identification of miRNAs and Their Target Genes Using Deep Sequencing and Degradome Analysis in Trifoliate Orange [Poncirus trifoliata L. Raf]
}

\author{
Jin-Zhi Zhang $\cdot$ Xiao-Yan Ai $\cdot$ Wen-Wu Guo • \\ Shu-Ang Peng $\cdot$ Xiu-Xin Deng $\cdot$ Chun-Gen Hu
}

Published online: 24 September 2011

(C) Springer Science+Business Media, LLC 2011

Erratum to: Mol Biotechnol

DOI 10.1007/s12033-011-9439-x

There is a spelling error in the title of the original publication. The correct title is "Identification of miRNAs and Their
Target Genes Using Deep Sequencing and Degradome Analysis in Trifoliate Orange [Poncirus trifoliata L. Raf]”.

The online version of the original article can be found under doi: 10.1007/s12033-011-9439-x.

J.-Z. Zhang · X.-Y. Ai · W.-W. Guo · S.-A. Peng ·

X.-X. Deng · C.-G. Hu $(\bowtie)$

Key Laboratory of Horticultural Plant Biology (Ministry of Education), College of Horticulture and Forestry Science, Huazhong Agricultural University, Wuhan 430070, China e-mail: chungen@mail.hzau.edu.cn

J.-Z. Zhang

e-mail: Jinzhizhang@webmail.hzau.edu.cn

X.-X. Deng

e-mail: xxdeng@mail.hzau.edu.cn

W.-W. Guo $\cdot$ X.-X. Deng

National Key Laboratory of Crop Genetic Improvement,

Huazhong Agricultural University, Wuhan 430070, China 